\title{
Use of Wavelets in Electrocardiogram Research: a Literature Review
}

\author{
Darwan $^{1}$, Hindayati Mustafidah ${ }^{2 *}$ \\ ${ }^{1}$ Mathematics Education, FITK, IAIN Syekh Nurjati Cirebon, Indonesia \\ ${ }^{2}$ Informatics Engineering, Universitas Muhammadiyah Purwokerto, Indonesia \\ ${ }^{1}$ darwanesyekhnurjati.ac.id \\ ${ }^{2 *}$ corr-author: h.mustafidah@ump.ac.id
}

\begin{abstract}
Currently the introduction and detection of heart abnormalities using electrocardiogram (ECG) is very much. ECG conducted many research approaches in various methods, one of which is wavelet. This article aims to explain the trends of ECG research using wavelet approach in the last ten years. We reviewed journals with the keyword title "ecg wavelet" and published from 2011 to 2020. Articles classified by the most frequently discussed topics include: datasets, case studies, pre-processing, feature extraction and classification/identification methods. The increase in the number of ECG-related articles in recent years is still growing in new ways and methods. This study is very interesting because only a few researchers focus on researching about it. Several approaches from many researchers are used to obtain the best results, both by using machine learning and deep learning. This article will provide further explanation of the most widely used algorithms against ECG research with wavelet approaches. At the end of this article, the critical aspects of ECG research can be done in the future, namely the use of data in the form of signals and images, as well as the use of deep learning to be a very large opportunity for researchers.
\end{abstract}

Keywords: ECG, wavelet, dataset, pre-processing, feature extraction

\section{INTRODUCTION}

Humans have several very important organs, one of which is the heart. Maintenance of human heart conditions is highly recommended, because this organ has a very vital role. Maintaining the condition and maintaining the heart early on is something very good. Because heart malfunction can result in sudden death. In recent years heart defects have still topped the list of deaths worldwide. Worldwide deaths are caused by heart defects [1]. Heart defects in 2019 are the cause of onethird of all deaths in the world, as well as the everincreasing number of deaths. Some of the countries with the highest number of deaths from heart disease in 2019 were China, followed by India, Russia, the United States, and Indonesia [2]. Therefore, we are expected to maintain the condition of the body, especially the heart in order to stay healthy.

World Health Organization (WHO) data in 2015 shows 17.7 million people worldwide die from cardiovascular disease (heart) or $45 \%$ of the 39.5 million deaths worldwide [3]. According to the AHA (America Heart Association) nearly one in three people die from heart disease. According to the Ministry of Health (2020) heart disease (especially coronary) is the highest cause of death, followed by cancer, diabetes mellitus with complications, tuberculosis, then chronic obstructive pulmonary disease (COPD).

To maintain the heart condition we are expected to always do a heart check. Heart condition checks are performed using aids such as: electrocardiogram (ECG), blood test, nuclear heart scanning, cardiac catheterization, cardiac training test, echocardiography and coronary angiography [4]. A frequent and common tool used to check for heart abnormalities is with an electrocardiogram (ECG) (Fig. 1).

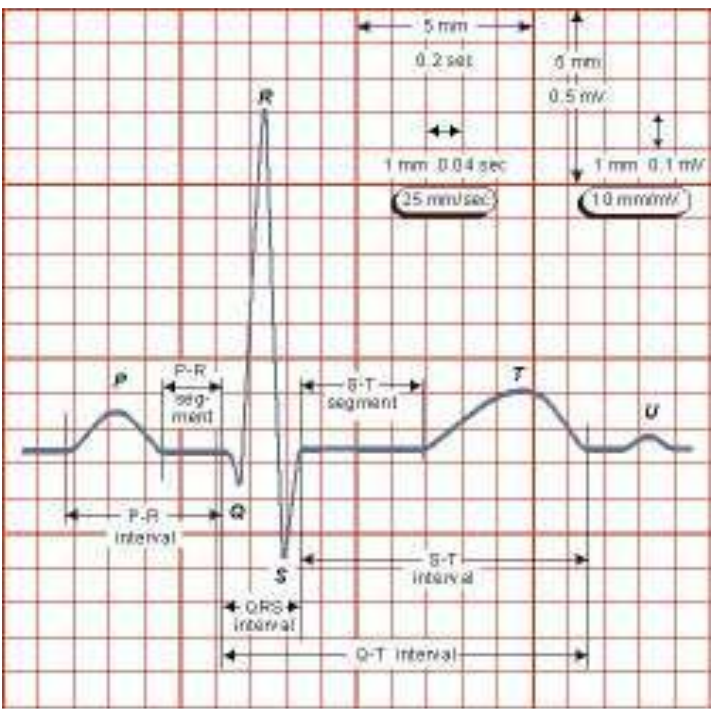

Fig. 1 Electrocardiogram [5] 
Because of the importance of keeping the heart condition healthy and controlled, there are many studies whose case studies use the heart. Many approaches are used to obtain good performance. So with the correct method of detecting this disease it is possible to apply a more directed treatment [1]. One approach that is often used is to use the wavelet method. Wavelet method is widely utilized in the pre-processing stage as well as feature extraction. This study tried to study literature related to ECG using wavelet approach within ten years (2011-2020). With the conduct of literature review is expected to obtain a road map related to future researches.

\section{METHOD}

Research related to electrocardiogram (ECG) has been widely done by many researchers. From the researcher's research, ECG research has been conducted by Pan and Tompkins [6]. Furthermore, many researchers who conducted ECG research also such as [7] whose research related to heart abnormalities arrhythmia using fuzzy logic method. Various method approaches are used to obtain a good end result, such as fuzzy, wavelet, machine learning or statistical methods [8]. There are even those who use tools (CSA) on Linux [9]. In this study, researchers tried to conduct research in terms of literature studies related to electrocardiograms (ECG).

Researchers began tracing literature studies related to journals that discussed ECG and used wavelet methods. ECG research using wavelets is spread in the journals of several disciplines such as computer science, electrical engineering, applied sciences, and others. Searches were conducted on several publication database sites published from 2011 to 2020 with the keyword "ECG Wavelet", and restricted to the field of Computer Science.

In this study, we looked at several related journals ("ecg wavelets") whose data was retrieved using data from Physionet and SID Medical Technology Co. Ltd.

\section{A. Physionet}

Physionet is one of the research resources for Physiological Signals established in 1999 under the auspices of the National Institutes of Health (NIH) as in Fig. 2. Physionet primary and ongoing mission is to catalyze research and education in the field of biomedical, by offering free access to physiological and clinical databases. So Physionet provides free datarelated access for research and educational purposes. Physionet also held a Computing in Cardiology conference, as well as an annual series of challenges, which focused its research on unsolved problems in the field of basic and clinical sciences. Members of the Physionet team are actively engaged in innovative work on physiological signal analysis, both from a basic and translation perspective. The Physionet platform is managed by members of the MIT Laboratory for Computational Physiology. Other core laboratories of Physionet Resources are Margaret and H.A. Rey Institute for Nonlinear Dynamics at Beth Israel Deaconess Medical Center. Some of the databases provided include MIT-BIH, Europe ST-Database, Fantasia Database, StPetersburg, Creighton University database (CUDB), PTB diagnostic ECG database, and others.

\section{B. SID Medical Technology Co. Ltd.}

Looking at its website SID Medical was founded in 2003, which is a medical technology company located in Shanghai (China) [11]. In December 2015, SID medical began offering a cloud-based ECG Application Program. With our PC and/or Mobile software installed in the Cloud. Each patient can upload ECG data and get an analysis report. Meanwhile, ECG specialists working with SID Medical can download and consult with patients

In the first stage of the document search results collected as many as 97 that researchers obtained. The second stage of 97 documents that match the criteria sought (ECG with wavelet method) there are 38 documents. The distribution of the appropriate article search results (38 articles) can be seen in Fig.3. So at this last stage, researchers analyzed the contents of each document based on a predetermined classification scheme.

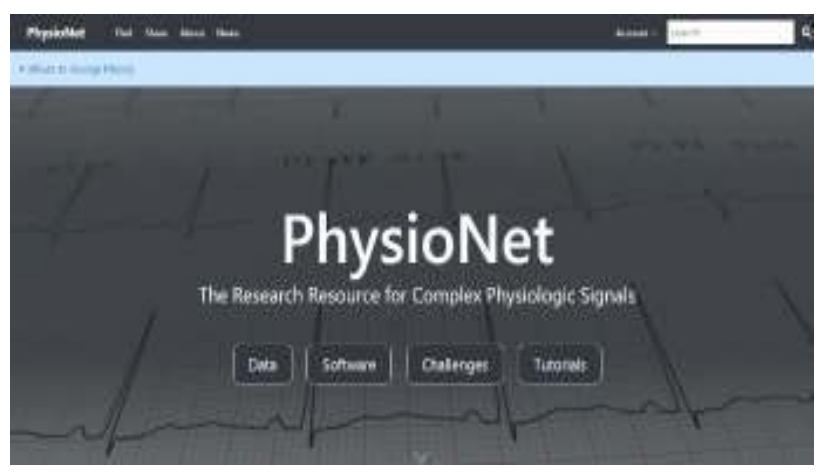

Fig. 2 Physionet website [10] 


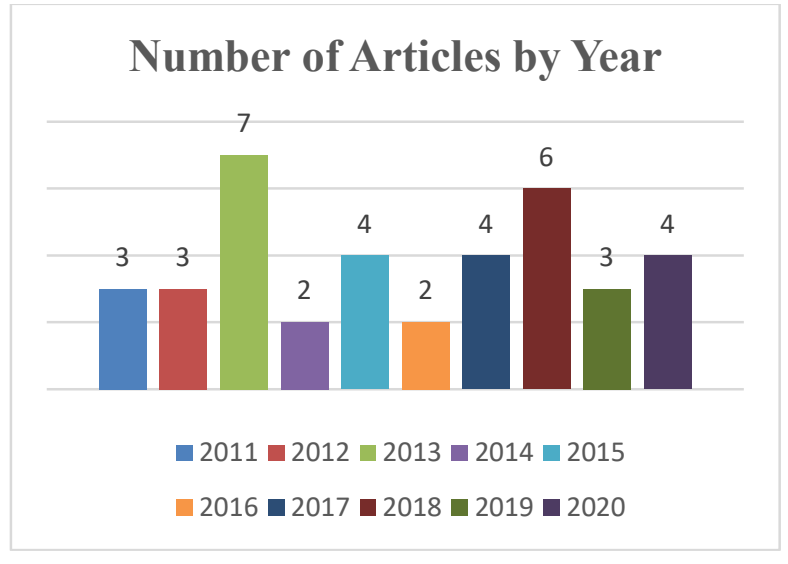

Fig. 3 number of articles by year

From several case studies that are often examined in research in the case of ECG and wavelets mostly based on the paper obtained that is related to Arrhythmia. From studies using arrhythmia case studies in general there are 10 studies [12-13], [16-17], [21],[25-26],[28],[38],[43]. Atrial fibrilation mentioned specifically there are four studies [27] [36],[46],[48]. Using normal data there are three studies [16],[39],[42]. Ventricular Tachyarrhytmia there are two studies [16],[37]. Other researches include Peak Detection R [15], Intracardiac Atrial Fibrillation (IAF) and Myaocard Infarct [16], Ischemia [18], Atherosclerosis [24], Atrial Fibrillation-Ventricular Fibrillation-Ventricular Contraction [32], Coronary Artery Disease [33], Premature Ventricular Contraction and Trial Premature Contraction [42], and ECG signal denoising [45].

\section{RESULTS AND DISCUSSION}

Systematically this study focused on 38 journals starting from 2011 to 2020 related to ECG using wavelets from both pre-processing, feature extraction and classification.

\section{A. Pre-Processing}

ECG related research mostly goes through several stages, namely pre-processing, character extraction and classification/identification. While the use of wavelets can be used at the pre-processing stage and most are also used at the stage of feature extraction. Some even do not go through the pre-processing stage and go directly to the characteristic extract. Wavelet methods used in the preprocessing phase based on the journals used are Wavelet Transform [12],[42],[47], Wavelet Decomposition [14], Discrete Wavelet Transform-DWT [19],[22],[34-37], [43-44], [1], Transform-WPT Package Wavelet [46]. As for pre-processing that does not use wavelets and uses other methods (Table I).

\section{B. Feature Extraction}

The extraction phase of this feature is one of the stages that is often used in research related to Artificial Intelligence. Related literature review of this study almost $67 \%$ paper used all using wavelet at the extraction stage of its feature. With the use of Discrete Wavelet Transform-DWT method consists of 50\% journals used using such methods include: [15], [18], [20], [24-25], [28-32], [35], [38], and [41]. Continuous Wavelet Transform (CWT) include: [17], [21], [23], and [39-40]. Wavelet Packet Decomposition (WPD): [33], [43], and [48]. Wavelet Packet Transform (WPT): [26], and Wavelet Reconstruction [41]. Some researchers are using methods other than Wavelet at the extraction stage of the feature as seen in Table II.

TABLE I

USE OF PRE-PROCESSING OTHER THAN WAVELETS

\begin{tabular}{lcc}
\hline \multicolumn{1}{c}{ Pre-Processing } & Years & Author \\
\hline Baseline Wander Removal & 2011 & {$[13]$} \\
QRS Complex Detection & 2012 & {$[15]$} \\
Spectral Analysis, Baseline Wander & & \\
Removing & 2012 & {$[16]$} \\
Segmentation & 2012 & {$[17]$} \\
QRS Subtraction & 2013 & {$[18]$} \\
Hidden Markov Model & 2013 & {$[20]$} \\
Pattern Identification (Local \& Global & & \\
Pattern) & 2013 & {$[21]$} \\
PCA & 2013 & {$[24]$} \\
Filtering raw signal and extraction of & & \\
beats & 2014 & {$[25]$} \\
P \& R peak detection & 2015 & {$[27]$} \\
Denoising using notch filter & 2015 & {$[29]$} \\
Noise Repair & 2015 & {$[30]$} \\
Denoising & 2016 & {$[31]$} \\
Key Features and Frequencies & 2016 & {$[32]$} \\
Denoising & 2018 & {$[38]$} \\
Baseline Drift Removal and Denoising, & & \\
Noise Removal & 2018 & {$[39]$} \\
Data Denoising and Data Segmentation & 2018 & {$[40]$} \\
Noise Identification & 2018 & {$[41]$} \\
Mean & 2019 & {$[41]$} \\
\hline
\end{tabular}


TABLE II

FEATURE EXTRACTION METHODS

\begin{tabular}{|c|c|c|}
\hline Feature Extraction & Years & Author \\
\hline Beat Normalitation & 2013 & [19] \\
\hline $\begin{array}{l}\text { Peak R \& Interval RR } \\
\text { Thresholding and Run-Length }\end{array}$ & 2013 & {$[22]$} \\
\hline Encoding & 2017 & [34] \\
\hline $\begin{array}{l}\text { Recurrence quantification analysis } \\
\text { (RQA) }\end{array}$ & 2017 & {$[36]$} \\
\hline Segmentation & 2018 & {$[37]$} \\
\hline $\begin{array}{l}\text { Statistic (mean, standard deviation, } \\
\text { kurtosis and skewness) }\end{array}$ & 2018 & {$[42]$} \\
\hline P,Q,R,S,T Peak & 2019 & {$[43]$} \\
\hline Histogram & 2020 & {$[44]$} \\
\hline
\end{tabular}

\section{Classification \& Performance}

At this stage it is mostly related to classification or identification. This identification stage is used many methods include: Artificial Neural Network, Support Vector Machine, Probabilistic Neural Network, and Convolution Neural Network. The selection of applied methods is usually based on the use of data. The use of the data used will affect the selection of methods at this stage. Classification or identification stage in addition to being able to identify or classify data there are usually some researchers looking for performance. Performance that is often used include accuracy, sensitivity and specificity. In finding the level of accuracy, sensitivity and specificity by using confusion matrix [49]. The use of Matrix Confusion is one of the methods used to obtain evaluation results based on matrix tables [50].

TABLE III

MODEL CONFUSION MATRiX [50]

\begin{tabular}{ccc}
\hline \multirow{2}{*}{ Correct Classification } & \multicolumn{2}{c}{ Classified } \\
\cline { 2 - 3 } & + & - \\
\hline+ & $\mathrm{TP}$ & $\mathrm{FN}$ \\
- & $\mathrm{FP}$ & $\mathrm{TN}$ \\
\hline
\end{tabular}

Accuracy using (1), sensitivity to (2), and specificity with (3) [49]:

$$
\begin{aligned}
& \text { Accuracy }=\frac{T P+T N}{T P+T N+F P+F N} \times 100 \% \\
& \text { Sensitivity }=\frac{T P}{T P+F N} \times 100 \% \\
& \text { Specificity }=\frac{T N}{T N+F P} \times 100 \%
\end{aligned}
$$

where:

$$
\begin{aligned}
& \mathrm{TP}=\text { True Positive }, \\
& \mathrm{TN}=\text { True Negative }, \\
& \mathrm{FP}=\text { False Positive }, \text { and } \\
& \mathrm{FN}=\text { False Negative } .
\end{aligned}
$$

From the above exposure can be presented overall research related to ECG with wavelet approach, and the methods used and some of them list the results of performance can be seen in Table IV.

\section{CONCLUSION}

ECG case studies are very varied to be raised in research. The use of data used by some researchers uses data available from Physionet with its various data banks. In addition, many researchers are taking data directly from patients. Data processing in pre-processing is very diverse from each researcher, which surely the output of the data presented will be very useful on the extract of features. From the paper used in this literature review almost $79.5 \%$ use wavelets at the extracting stage of its characteristics, as well as wavelet methods used are also diverse. Variations in the use of machine learning algorithms can be used in the classification or identification stage such as Artificial Neural Network, Support Vector Machine, Probabilistic Neural Network, and Convolution Neural Network. Future Work research is very wide open related to electrocardiogram (ECG), both the use of data in the form of signals and images. ECG research using Deep Learning is still very little, so this is a very big opportunity for researchers in researching ECG using such methods. 
TABLE IV

ECG RESEARCH WITH WAVELETS APPROACH

\begin{tabular}{|c|c|c|}
\hline Years & $\begin{array}{c}\text { Approach } \\
\end{array}$ & The Highest Performance \\
\hline 2011 & $\begin{array}{l}\text { Wavelet Transform[12], Baseline Wander Removal; Fuzzy Wavelet } \\
\text { Learning Vector Quantization (FWLVQ) [13], Wavelet } \\
\text { Decomposition; Thresholding \& Wavelet Reconstruction [14] }\end{array}$ & $\begin{array}{l}\text { Sensitivity from MIT-BIH: } 99,77 \\
\% \text {; Sensitivity from Europe: } 99,81 \\
\% \text {; Accuracy: } 90,20 \%\end{array}$ \\
\hline 2012 & $\begin{array}{l}\text { QRS Complex Detection; DWT [15], Spectral Analysis, Baseline } \\
\text { Wander Removing; 6-level Wavelet Transformation Decomposition; } \\
\text { ANN Backpropagation [16], Segmentation; Continuous Wavelet } \\
\text { Transform (CWT); ANN Backpropagation [17] }\end{array}$ & $\begin{array}{l}\text { Accuracy: } 87,42 \% \text {; Sensitivity: } \\
87 \% \text {; Specificity: } 95 \%\end{array}$ \\
\hline 2013 & $\begin{array}{l}\text { QRS Subtraction; DWT \& Morpology Feature Calculation; SVM } \\
\text { [18], DWT; Beat Normalization; SVM [19], Hidden Markov Model; } \\
\text { Discrete Wavelet Transform (DWT) [20], Pattern Identification } \\
\text { (Local \& Global Pattern); Continuous Wavelet Transform (CWT) } \\
\text { [21], Discrete Denoised Wavelet; R Peak \& Interval RR [22], } \\
\text { Coefficient Wavelet Transform (CWT) - Morlet [23], PCA; Discrete } \\
\text { Wavelet Transform (DWT); SVM [24] }\end{array}$ & $\begin{array}{l}\text { Accuracy: 93,63 \%; Sensitivity: } \\
\text { 95,20\%; Specificity: } 93,29 \% \text {; } \\
\text { Accuracy Local Pattern 1: } 73,3 \\
\% \text {; Accuracy Local Pattern 2: } \\
\text { 75,0\%; } \\
\text { Accuracy Global Pattern: } 86,6 \% \text {; }\end{array}$ \\
\hline 2014 & $\begin{array}{l}\text { Filtering raw signal and extraction of beats; Discrete Wavelet } \\
\text { Transform (DWT); Multi-Level Perceptron NN [25], Wavelet Packet } \\
\text { Transform (WPT); Probabilistic Neural Network (PNN) [26] }\end{array}$ & Accuracy : 97,92\% \\
\hline 2015 & $\begin{array}{l}\text { P \& R peak detection; Stationary Wavelet Transformation; SVM } \\
\text { [27], Discrete Wavelet Transform (DWT) \& Dual Tree } \\
\text { Complex Wavelet Transform (DTCWT); Multi- } \\
\text { layer Backpropagation Neural Network [28], Denoising using notch } \\
\text { filter; Discrete Wavelet Transform (DWT); Statistic (mean, variance, } \\
\text { standard deviation, entropy) [29], Noise Repair; DWT-Daubechies } \\
\text { [30] }\end{array}$ & $\begin{array}{l}\text { Sensitivity: } 97,00 \% \text {; Specificity : } \\
\text { 97,10\%; Sensitivity DWT: } 91,23 \\
\% \text {; Sensitivity DTCWT: } 91,23 \%\end{array}$ \\
\hline 2016 & $\begin{array}{l}\text { Denoising; Discrete Wavelet Transform (DWT) [31], Key Features } \\
\text { and Frequencies; Discrete Wavelet Transformation; SVM [32] }\end{array}$ & Accuracy : 96,89\% \\
\hline 2017 & $\begin{array}{l}\text { Wavelet Packet Decomposition (WPD) Level 4; K-Nearest } \\
\text { Neighbors (KNN) [33], Discrete Wavelet Transformation (DWT); } \\
\text { Thresholding and Run-Length Encoding [34], DWT (Sym8 \& } \\
\text { Coeiflet4); Thresholding - Invers DWT [35], Discrete Wavelet } \\
\text { Transform (DWT); Recurrence quantification analysis (RQA); } \\
\text { Probabilistic Neural Network (PNN) [36] }\end{array}$ & $\begin{array}{l}\text { Accuracy: } 100 \% \text {; Sensitivity : } \\
99,64 \% \text {; Specificity : } 99,70 \%\end{array}$ \\
\hline 2018 & $\begin{array}{l}\text { Daubechies wavelet } 6 \text { (db6); Segmentation; Eleven Layers Of } \\
\text { Convolutional Neural Network (CNN) [37], Denoising; Discrete } \\
\text { Wavelet Transform; Wavelet Network Classifier [38], Baseline Drift } \\
\text { Removal and Denoising, Noise Removal; Continuous Wavelet } \\
\text { Transform (CWT) and Wavelet Analysis [39], Data Denoising and } \\
\text { Data Segmentation; CWT; CNN [40], Noise Identification; Inverse } \\
\text { Discrete Wavelet Transform (IDWT) - Sym8 [41], Transformasi } \\
\text { Wavelet - Haar; Statistic (mean, standard deviation, kurtosis and } \\
\text { skewness); Random Forest and information gain analysis [42] }\end{array}$ & $\begin{array}{l}\text { Accuracy: } 99,23 \% \text {; Sensitivity: } \\
\text { 99,41 \%; Specificity: } 98,91 \% \text {; } \\
\text { Accuracy Normal: } 84 \% \text {; } \\
\text { Accuracy PVC: } 98 \% \text {; Accuracy } \\
\text { APC: } 86 \%\end{array}$ \\
\hline 2019 & $\begin{array}{l}\text { DWT db8 Decomposition Level 8; P,Q,R,S,T Peak; Intelligence } \\
\text { Decision Rule [43], Wavelet Decomposition; Coefficient Processing; } \\
\text { Wavelet Reconstruction [44], Mean; Wavelet Decomposition - Haar } \\
\text { \& Symlet [45] }\end{array}$ & Sensitivity : 99,58\% \\
\hline 2020 & $\begin{array}{l}\text { (DWT) Wavelet Based on Atomic Function \& Z-Score; Decision } \\
\text { Fine Tree [45], Wavelet Packet Transform (WPT); Histogram; ANN } \\
\text { [46], Wavelet Transform (Comparison 14 Wavelet) [47]; Wavelet } \\
\text { Decomposition - daubechies; Convolution Neural Network [48] }\end{array}$ & $\begin{array}{l}\text { Accuracy: } 98,90 \% \text {; Sensitivity: } \\
\text { 98,70\%; Specificity: } 98,90 \%\end{array}$ \\
\hline
\end{tabular}

\section{REFERENCES}

[1] A. Hernandez-matamoros and H. Fujita, "ScienceDirect
Recognition of ECG signals using wavelet based on atomic functions," Integr. Med. Res., pp. 1-12, 2020, doi: 10.1016/j.bbe.2020.02.007. 
[2] Gading and Anna, "Penyakit Jantung Penyebab Kematian Utama di Dunia Artikel ini telah tayang di Kompas.com dengan judul 'Penyakit Jantung Penyebab Kematian Utama di Dunia', Klik untuk baca: https://ifestyle.kompas.com/read/2020/12/14/10160752 0/penyakit-jantung-penyebab-ke," Kompas.com. https://lifestyle.kompas.com/read/2020/12/14/10160752 0/penyakit-jantung-penyebab-kematian-utama-didunia?page=all (accessed Jan. 26, 2021).

[3] Biro Komunikasi dan Pelayanan Masyarakat, "Penyakit Tidak Menular Kini Ancam Usia Muda," Kementeri. Kesehat. RI, pp. 7-9, 2020, [Online]. Available: https://www.kemkes.go.id/article/view/20070400003/pe nyakit-tidak-menular-kini-ancam-usia-muda.html.

[4] J. Y. J. K. U. Kang and Y. Lee, "Coronary heart disease optimization system on adaptive-network- based fuzzy inference system and linear discriminant analysis ( ANFIS - LDA )," 2013, doi: 10.1007/s00779-0130737-0.

[5] P. Siwindarto, "Electrocardiograph (ECG / EKG)," Brawijaya.

http://instrumentasi.lecture.ub.ac.id/electrocardiograph/ (accessed Mar. 10, 2021).

[6] J. Pan and W. J. Tompkins, "A Real-Time QRS Detection Algorithm," IEEE Trans. Biomed. Eng., vol. BME-32, no. 3, pp. 230-236, 1985, doi: 10.1109/TBME.1985.325532.

[7] J. Cabello, D.; Barra, S.; Ruiz, R.; Zapata, E.L.; and Mira, "FUZZY CLUSTERING: APPLICATION TO THE DIAGNOSIS OF VENTRICULAR ARRYTHMIAS," IEEE Eng. Med. Biol. Soc. 10TH Annu. Int. Conf., no. Cardiac Arrhythmia Analysis, 1988, doi: CH25668/88/0000--0005.

[8] A. Minchóle, F. Jager, and P. Laguna, "Discrimination between demand and supply ischemia episodes in holter recordings," Annu. Int. Conf. IEEE Eng. Med. Biol. Proc., pp. 2579-2582, 2007, doi: 10.1109/IEMBS.2007.4352856.

[9] T. H. Tan, C. S. Chang, Y. F. Chen, and C. Lee, "Implementation of a portable personal EKG signal monitoring system," Lect. Notes Comput. Sci. (including Subser. Lect. Notes Artif. Intell. Lect. Notes Bioinformatics), vol. 4901 LNCS, pp. 122-128, 2008, doi: 10.1007/978-3-540-77413-6_16.

[10] Physionet, "Physionet," 1970. https://physionet.org/ (accessed Mar. 10, 2021).

[11] S. Medical, "SID Medical," 2003. http://www.sidmedical.cn/en/index.asp (accessed Mar. 10, 2021).

[12] L. Y. Di Marco and L. Chiari, "A wavelet-based ECG delineation algorithm for 32-bit integer online processing," Biomed. Eng. Online, vol. 10, pp. 1-19, 2011, doi: 10.1186/1475-925X-10-23.

[13] T. Imah, E. M., and Basaruddin, "KLASIFIKASI BEAT ARITMIA PADA SINYAL EKG MENGGUNAKAN
FUZZY WAVELET LEARNING VECTOR QUANTIZATION," J. Ilmu Komput. dan Inf., vol. 4, no. $1,2011$.

[14] B. S. Widodo, "Aplikasi Wavelet Untuk Penghilangan Derau Isyarat Elektrokardiograf," J. Buana Inform., vol. 2, no. 2, pp. 95-101, 2011, doi: 10.24002/jbi.v2i2.312.

[15] A. Mukherjee and K. K. Ghosh, "An efficient wavelet analysis for ECG signal processing," 2012 Int. Conf. Informatics, Electron. Vision, ICIEV 2012, pp. 411-415, 2012, doi: 10.1109/ICIEV.2012.6317419.

[16] A. Surtono, T. S. Widodo, and M. Tjokronagoro, "Analisis Klasifikasi Sinyal EKG Berbasis Wavelet dan Jaringan Syaraf Tiruan,” vol. 1, no. 3, pp. 60-66, 2012.

[17] F. Lutfi and A. Arifin, "Klasifikasi Sinyal Elektrokardiografi Menggunakan Wavelet Transform dan Neural Network," 13th Semin. Intell. Technol. Its Appl., vol. 62, no. 62 31, pp. 136-140, 2012.

[18] C. H. Fan, Y. Hsu, S. N. Yu, and J. W. Lin, "Detection of myocardial ischemia episode using morphological features," Proc. Annu. Int. Conf. IEEE Eng. Med. Biol. Soc. EMBS, pp. 7334-7337, 2013, doi: 10.1109/EMBC.2013.6611252.

[19] Q. Zheng et al., "A novel multi-resolution SVM (MRSVM) algorithm to detect ECG signal anomaly in WECARE project," ISSNIP Biosignals Biorobotics Conf. BRC, 2013, doi: 10.1109/BRC.2013.6487453.

[20] N. P. Hughes, L. Tarassenko, and S. J. Roberts, "Markov models for automated ECG interval analysis," $A d v$. Neural Inf. Process. Syst., 2004.

[21] K. Balasundaram, S. Masse, K. Nair, and K. Umapathy, "Automated signal pattern detection in ECG during human ventricular arrhythmias," Proc. Annu. Int. Conf. IEEE Eng. Med. Biol. Soc. EMBS, pp. 1029-1032, 2013, doi: 10.1109/EMBC.2013.6609679.

[22] J. Utama, "Electrocardiogram ( ECG ) dengan Noise Reduction Berbasis Wavelet Menggunakan Pemrograman LabVIEW Electrocardiogram ( ECG ) with Noise Reduction Based on Wavelet Using LabVIEW Programming," vol. 1, no. 1, pp. 40-45, 2013.

[23] S. Barmase, S. Das, and S. Mukhopadhyay, "Wavelet Transform-Based Analysis of QRS complex in ECG Signals," 2013, [Online]. Available: http://arxiv.org/abs/1311.6460.

[24] A. Kaveh and W. Chung, "Automated classification of coronary atherosclerosis using single lead ECG," 2013 IEEE Conf. Wirel. Sensor, ICWISE 2013, pp. 108-113, 2013, doi: 10.1109/ICWISE.2013.6728790.

[25] P. Sarma, S. R. Nirmala, and K. K. Sarma, "ECG classification using wavelet subband energy based features," 2014 Int. Conf. Signal Process. Integr. Networks, SPIN 2014, pp. 785-790, 2014, doi: 10.1109/spin.2014.6777061.

[26] K. Daqrouq, A. Alkhateeb, M. N. Ajour, and A. Morfeq, "Neural network and wavelet average framing 
percentage energy for atrial fibrillation classification," Comput. Methods Programs Biomed., vol. 113, no. 3, pp. 919-926, 2014, doi: 10.1016/j.cmpb.2013.12.002.

[27] S. Asgari, A. Mehrnia, and M. Moussavi, "Automatic detection of atrial fibrillation using stationary wavelet transform and support vector machine," Comput. Biol. Med., vol. 60, pp. 132-142, 2015, doi: 10.1016/j.compbiomed.2015.03.005.

[28] M. Thomas, M. K. Das, and S. Ari, "Automatic ECG arrhythmia classification using dual tree complex wavelet based features," AEU - Int. J. Electron. Commun., vol. 69, no. 4, pp. 715-721, 2015, doi: 10.1016/j.aeue.2014.12.013.

[29] A. Sharmila, P. Mahalakshmi, V. N. V. L. N. G. Sharma, G. Dey, and K. Yashwanth, "Detection of artial fibrillation disorder by ecg using discrete wavelet transforms," Asian J. Pharm. Clin. Res., vol. 8, no. 6, pp. 4-6, 2015.

[30] H. AGUIRRE-RAMOS, "Wavelets for correction of ECG images," ECORFAN J., vol. 1, no. 1, pp. 36-41.

[31] A. Sampath and T. R. Sumithira, "ECG Morphological Marking using Discrete Wavelet Transform," Intell. Decis. Technol., vol. 10, no. 4, pp. 373-383, 2016, doi: 10.3233/IDT-160264.

[32] H. Ibrahim and I. Owis Mohamed, “Automatic arrhythmia detection using support vector machine based on discrete wavelet transform," J. Med. Imaging Heal. Informatics, vol. 6, no. 1, pp. 204-209, 2016, doi: 10.1166/jmihi.2016.1611.

[33] S. L. Oh et al., "Automated identification of coronary artery disease from short-term 12 lead electrocardiogram signals by using wavelet packet decomposition and common spatial pattern techniques," J. Mech. Med. Biol., vol. 17, no. 7, 2017, doi: 10.1142/S0219519417400073.

[34] H. Myo Tun, "Analysis on ECG Data Compression Using Wavelet Transform Technique," Int. J. Psychol. Brain Sci., vol. 2, no. 6, p. 127, 2017, doi: 10.11648/j.ijpbs.20170206.12.

[35] A. Aqil, M.; Jbari, A.; Bourouhou, "ECG Signal Denoising by Discrete Wavelet Transform," iJOE, vol. 13, no. 9, pp. 51-68, 2017.

[36] S. Saraswat, G. Srivastava, and S. Shukla, "Wavelet transform based feature extraction and classification of atrial fibrillation arrhythmia," Biomed. Pharmacol. J., vol. 10, no. 4, pp. 1715-1725, 2017, doi: $10.13005 / \mathrm{bpj} / 1284$.

[37] U. R. Acharya et al., "Automated identification of shockable and non-shockable life-threatening ventricular arrhythmias using convolutional neural network," Futur. Gener. Comput. Syst., vol. 79, no. August, pp. 952-959, 2018, doi: 10.1016/j.future.2017.08.039.

[38] D. D. Patil and R. P. Singh, "ECG Classification Using
Wavelet Transform and Wavelet Network Classi fi er," pp. 289-303, doi: 10.1007/978-981-10-7868-2.

[39] A. Rodríguez Gómez and Á. Jiménez-Casas, “Analysis of the ECG Signal Recognizing the QRS Complex and P and T Waves, Using Wavelet Transform," Am. J. Eng. Res. (AJER, no. 7, pp. 51-59, 2018, [Online]. Available: www.ajer.org.

[40] R. He et al., "Automatic detection of atrial fibrillation based on continuous wavelet transform and 2D convolutional neural networks," Front. Physiol., vol. 9, no. AUG, pp. 1-11, 2018, doi: 10.3389/fphys.2018.01206.

[41] B. R. de Oliveira, M. A. Q. Duarte, C. C. E. de Abreu, and J. V. Filho, "A wavelet-based method for power-line interference removal in ECG signals," Res. Biomed. Eng., vol. 34 , no. 1 , pp. $73-86$, 2018, doi: 10.1590/24464740.01817.

[42] Y. V. Saragih, A. W. Widodo, and M. A. Rahman, "Pemilihan Fitur Berbasis Wavelet untuk Klasifikasi Denyut Jantung dari Rekaman Elektrokardiogram," vol. 3, no. 4, pp. 3140-3147, 2019.

[43] M. Arumugam and A. K. Sangaiah, "Arrhythmia identification and classification using wavelet centered methodology in ECG signals," Concurr. Comput. , vol. 32, no. 17, pp. 1-17, 2020, doi: 10.1002/cpe.5553.

[44] Z. Wang, J. Zhu, T. Yan, and L. Yang, "A new modified wavelet-based ECG denoising," Comput. Assist. Surg., vol. 24, no. sup1, pp. 174-183, 2019, doi: 10.1080/24699322.2018.1560088.

[45] D. Zhang et al., "An ECG signal de-noising approach based on wavelet energy and sub-band smoothing filter," Appl. Sci., vol. 9, no. 22, 2019, doi: 10.3390/APP9224968.

[46] J. Wang, P. Wang, and S. Wang, "Biomedical Signal Processing and Control Automated detection of atrial fibrillation in ECG signals based on wavelet packet transform and correlation function of random process," Biomed. Signal Process. Control, vol. 55, p. 101662, 2020, doi: 10.1016/j.bspc.2019.101662.

[47] C. C. Chen and F. R. Tsui, "Comparing different wavelet transforms on removing electrocardiogram baseline wanders and special trends," BMC Med. Inform. Decis. Mak., vol. 20, 2020, doi: 10.1186/s12911-020-01349-x.

[48] Y. Zhao, J. Cheng, P. Zhang, and X. Peng, "ECG classification using deep CNN improved by wavelet transform," Comput. Mater. Contin., vol. 64, no. 3, pp. 1615-1628, 2020, doi: 10.32604/cmc.2020.09938.

[49] J. Han, Jiawei; Kamber, Micheline; Pei, Data Mining Concepts and Techniques, Third. Morgan Kaufmann Publishers, 2012.

[50] M. Bramer, Principles of Data Mining, Second. Springer, 2013. 
JUITA: Jurnal Informatika e-ISSN: 2579-8901; Vol. 9, No. 1, May 2021 